\title{
ESTUDO DA VIDA-DE-PRATELEIRA DE FRUTA ESTRUTURADA E DESIDRATADA OBTIDA DE POLPA CONCENTRADA DE MAMÃO ${ }^{1}$
}

\author{
Regina Kitagawa GRIZOTTO ${ }^{2, *}$, Shirley Aparecida Garcia BERBARI², \\ Sílvia Cristina Sobottka Rolim de MOURA², Melissa Lima CLAUS ${ }^{3}$
}

\begin{abstract}
RESUMO
A fruta estruturada contendo polpa concentrada de mamão (700 g/kg), sacarose ( $340 \mathrm{~g} / \mathrm{kg})$, alginato de sódio (7 g/kg), pectina de baixa metoxilação $\left(7 \mathrm{~g} / \mathrm{kg}\right.$ ) e glicerol ( $50 \mathrm{~g} / \mathrm{kg}$ ), cortada no formato cilindro sólido (vol. aprox. $2 \mathrm{~cm}^{3}$ ), desidratada ( $12 \%$ umidade), com cobertura de açúcar impalpável, foi utilizada no estudo de vida-de-prateleira. Foram realizadas análises físicas, químicas, sensoriais e microbiológicas no produto devidamente acondicionado em sacos de filme multilaminado, para acompanhar a evolução da perda de qualidade do produto armazenado a 0,25 e $35^{\circ} \mathrm{C}$, durante 120 dias. Os resultados mostraram que a firmeza objetiva e as avaliações subjetivas da textura e qualidade global, foram as que melhor identificaram a queda na qualidade da fruta estruturada durante a estocagem. Na condição acelerada $\left(35^{\circ} \mathrm{C}\right)$, o produto foi considerado próprio para consumo até 56 dias de armazenamento, quando atingiu a nota 4,5 (não gostei/nem desgostei) da escala de qualidade global do teste sensorial. Os dados cinéticos, $\mathrm{E}_{\mathrm{a}}=19,89 \mathrm{kcal} / \mathrm{mol}$ e $\mathrm{Q}_{10}=3$, estabelecidos no teste acelerado, permitiram estimar o limite da vida útil em 168 dias para o produto armazenado a $25^{\circ} \mathrm{C}$. Em condições normais de armazenamento, estimam-se 154 dias de vida útil com base na equação da qualidade global dos resultados sensoriais.
\end{abstract}

Palavras-chave: estabilidade, polpa de frutas, texturização, avaliação sensorial.

\section{SUMMARY}

SHELF LIFE STUDIES OF RESTRUCTURED AND DRIED FRUIT MADE FROM CONCENTRATED PAPAYA PULP. The restructured fruit made with concentrated papaya pulp (700 g/kg), sugar (340 g/ kg), sodium alginate (7 g/kg), low methoxy pectin $(7 \mathrm{~g} / \mathrm{kg})$ and glycerol (50 g/kg), cut in a solid cylindrical form (volume approx. $\left.2 \mathrm{~cm}^{3}\right)$, dehydrated ( $12 \%$ humidity) and covered with icing sugar, was chosen for the shelf life studies. The physical, chemical, sensory and microbiological analyses were made on the product packaged in a multi layer film and stored at 0,25 and $35{ }^{\circ} \mathrm{C}$, for 120 days to check for quality loss. The results showed that the firmness (TA.XT2 Texturometer) and the subjective evaluations of texture and overall quality were the evaluation that best explained the quality loss of the restructured fruit during storage. Under accelerated conditions $\left(35^{\circ} \mathrm{C}\right)$ the product was considered adequate for consumption for up to 56 days, when it reached 4.5 (neither liked nor disliked) from the overall quality scale of the sensorial test. The kinetic values, $\mathrm{E}_{\mathrm{a}}=19,89 \mathrm{kcal} / \mathrm{mol}$ and $\mathrm{Q}_{10}=3$, established using accelerated shelf life testing, allowed for the estimation of a shelf life of 168 days for the product at $25^{\circ} \mathrm{C}$. Under normal storage conditions, the shelf life of the product being estimated as 154 days based on the overall quality using the sensory results.

Keywords: stability, fruit pulp, texturization, sensorial evaluation.

\section{1 - INTRODUÇÃO}

A fruta estruturada pode ser considerada um exemplo da industrialização de matérias-primas de baixo custo, oriundas de frutas que se encontram fora de classificação para comercialização no mercado "in natura", bem como de excedentes de produção durante o período de safra. Esses produtos requerem um agente texturizante, geralmente o alginato puro ou em mistura com pectina, para controlar as propriedades funcionais. A fruta estruturada pode ser utilizada na formulação de produtos de confeitaria, alimentos congelados ou consumida na forma em que se apresenta,

\footnotetext{
${ }^{1}$ Recebido para publicação em 28/12/2005. Aceito para publicação em 6/7/2006 (001658)

${ }^{2}$ Centro de Pesquisa e Desenvolvimento de Hortifrutícolas,

Instituto de Tecnologia de Alimentos,

Av. Brasil, 2880, C. P. 139, CEP 13070-178, Campinas (SP),

E-mail: regina@ital.sp.org.br

${ }^{3}$ Bolsista de Iniciação Científica/CNPq,

Faculdade de Engenharia de Alimentos,

Universidade Estadual de Campinas,

C. P. 6177, CEP 13083-970, Campinas (SP)

* A quem a correspondência deve ser enviada
}

como um confeito, similarmente às barras de frutas (fruit bar), também conhecidas como couro de frutas (leather fruit) [16].

O procedimento tecnológico para produção da fruta estruturada contendo teor elevado de polpa de mamão e hidrocolóides (alginato e pectina de baixa metoxilação) foi recentemente estabelecido por GRIZOTTO et al. [4]. Por se tratar de um produto novo, torna-se necessário um estudo para identificar as reações de deterioração, às quais o produto está sujeito em função das condições ambientais, e estabelecer quanto da qualidade inicial pode ser perdida antes do produto ser considerado inadequado para o consumo humano. Dentre os fatores ambientais que contribuem na perda da qualidade para a maioria dos alimentos, a exposição a temperaturas elevadas é a mais importante. Quanto maior a temperatura, maior é a perda da qualidade do alimento [6, 7].

A vida-de-prateleira de produtos pode ser definida como um período de armazenamento em que produtos com alta qualidade inicial permanecem adequados para consumo [18]. Entretanto, como os mecanismos de perda de quali- 
dade dos alimentos são complexos e os consumidores têm sensibilidade diferente a essa perda, é impossível estabelecer uma definição universal de vida-de-prateleira. De acordo com MORI [8], o estudo de vida-de-prateleira de produtos alimentícios consiste em submeter várias amostras a uma série de testes e examiná-las durante um período de tempo até o limite de aceitação. São observadas as alterações na qualidade do produto e o tempo que ele leva para se deteriorar até o limite que o torna impróprio para o consumo. A identificação dos atributos que se alteram e a definição quantitativa desse atributo são maneiras de monitorar a perda de qualidade durante o armazenamento [10].

O uso de Testes Acelerados de Vida-de-Prateleira (TAVP) permite, em um tempo de estudo reduzido, estimar a vidade-prateleira do produto de interesse, em diferentes condições de armazenamento. Com base no modelo de Arrhenius [6] podem-se calcular os parâmetros cinéticos, energia de ativação (Ea) e o fator de aceleração da temperatura $\left(Q_{10}\right)$, os quais descrevem quão rapidamente uma reação irá ocorrer, se um produto for mantido a uma outra temperatura, incluindo as temperaturas de abuso. Se o valor de $Q_{10}$ for conhecido, ele pode então ser usado na extrapolação da vida-de-prateleira para temperaturas inferiores, tais como aquelas encontradas durante a comercialização. VITALI et al. [17] e NETTO [10] salientam, face aos erros e aproximações a que estão sujeitos os métodos acelerados, a necessidade da confirmação da vida-de-prateleira em condições normais de armazenamento.

O objetivo deste trabalho foi estabelecer a vida-de-prateleira da fruta estruturada de polpa concentrada de mamão e avaliar as reações de transformações físicas, químicas e sensoriais nas amostras armazenadas sob três condições de temperatura $\left(0,25,35^{\circ} \mathrm{C}\right)$, e identificar, dentre essas reações, aquelas que mais afetam a qualidade do produto.

\section{2 - MATERIAL E MÉTODOS}

\section{1 - Fruta estruturada de polpa de mamão concentrada}

A fruta estruturada utilizada no estudo de vida-de-prateleira foi produzida a partir de polpa de mamão concentrada e demais ingredientes listados na Tabela 1, de acordo com o procedimento estabelecido por GRIZOTTO et al. [4].

TABELA 1 - Fruta estruturada de polpa concentrada de mamão - Formulação

\begin{tabular}{lc}
\hline \multicolumn{1}{c}{ Ingrediente } & Quantidade (g/kg) \\
\hline Polpa de mamão concentrada & 696,58 \\
(pH 4,10, 20 ${ }^{\circ}$ Brix) & \\
Açúcar & 342,00 \\
Alginato & 7,00 \\
Pectina baixa metoxilação & 7,00 \\
Glicerol & 50,00 \\
$\mathrm{CaHPO}_{4}$ & 4,00 \\
$\mathrm{NaHCO}_{3}$ & 2,25 \\
Glucona delta lactona & 10,00 \\
Água & 13,33 \\
\hline
\end{tabular}

A polpa de mamão obtida de aproximadamente $180 \mathrm{~kg}$ de mamão in natura, no estádio de maturação $3 / 4$ madura e níveis adequados de pectina ( $0,9 \%)$, sólidos solúveis ( $\left.11,6{ }^{\circ} \mathrm{Brix}\right) \mathrm{e}$ pigmentos vermelhos $(\mathrm{a}=+10,48)$, foi concentrada em tacho encamisado (Groen MGF Co., EUA), capacidade para $30 \mathrm{~L}$, operando a $40-45{ }^{\circ} \mathrm{C}$ sob vácuo ( $88 \mathrm{kPa}$ ). O nível máximo de concentração para polpa de mamão foi estabelecido em $20{ }^{\circ}$ Brix por GRIZOTTO et al. [3], com base na manutenção das características originais da polpa, em especial os componentes aromáticos responsáveis pelo sabor.

O glicerol (50 g/kg) foi adicionado à polpa concentrada, com base no nível máximo permitido para balas e produtos similares descrito por ANTUNES \& CANHO [1], e, em função do teor de sólidos solúveis determinado, calculou-se a quantidade de sacarose suficiente para elevar o teor de sólidos solúveis até $50{ }^{\circ} \mathrm{Brix}$. O objetivo principal do glicerol foi abaixar a atividade de água, mas também promover aumento em $20 \%$ no teor de sólidos solúveis da polpa, contribuindo na redução da quantidade de sacarose necessária para atingir $50{ }^{\circ} \mathrm{Brix}$. Os coadjuvantes tecnológicos fosfato de cálcio bibásico anidro $\left(\mathrm{CaHPO}_{4}\right)$, bicarbonato de sódio $\left(\mathrm{NaHCO}_{3}\right)$ e glucona delta lactona foram adicionados com os respectivos objetivos de fornecer cálcio, ajustar a acidez da polpa de mamão e abaixar o pH do produto após a estruturação. A estruturação foi conduzida na polpa previamente aquecida a $60{ }^{\circ} \mathrm{C}$, em banho-maria. MOUQUET et al. [9] demonstrou ser fundamental o pré-aquecimento da polpa a $60{ }^{\circ} \mathrm{C}$ para que ocorra a gelificação da mistura. À polpa foi adicionada uma mistura seca de hidrocolóides, alginato e pectina, dispersos em sacarose, sob agitação (390 rpm), no Misturador-Desaerador (Cunnington \& Cooper Ltda., England), operando sob vácuo (88 kPa). Após dez minutos de agitação, o sistema foi aberto, liberando o vácuo, sendo adicionadas as suspensões de cálcio insolúvel (4 g/kg) e glucona delta lactona (10 g/kg), recém preparadas em aproximadamente $6 \mathrm{~mL}$ de água destilada. Além da água utilizada no preparo das suspensões, nenhuma outra foi adicionada. Os ingredientes foram então vigorosamente homogeneizados sob vácuo no Misturador-Desaerador por mais $5 \mathrm{~min}$. A moldagem da fruta estruturada foi realizada em placas de Petri de $50 \mathrm{~mm}$ de diâmetro e $10 \mathrm{~mm}$ de altura, com capacidade para $25 \mathrm{~g}$ da amostra, aproximadamente. $\mathrm{O}$ material moldado foi mantido sob refrigeração a $10{ }^{\circ} \mathrm{C}$ durante $24 \mathrm{~h}$, para completar a gelificação.

A fruta estruturada destinada à avaliação sensorial foi cortada no formato cilindro sólido, volume aproximado de $2 \mathrm{~cm}^{3}$ e submetida à secagem a $60{ }^{\circ} \mathrm{C}$ em câmara com circulação de ar, até umidade final de $12 \%$ e, posteriormente, coberta com camada de açúcar impalpável, com o objetivo de reduzir a água livre e minimizar a adesividade excessiva na superfície da fruta estruturada.

\section{2 - Acompanhamento da vida-de-prateleira}

Aproximadamente 36 unidades de fruta estruturada no formato cilindro sólido, volume aproximado de $2 \mathrm{~cm}^{3}$, foram acondicionadas em sacos de filme multilaminado da CRYOVAC (Duncan, USA), dimensão 0,018 m², com baixa 
permeabilidade ao vapor d'água (6 g água $/ \mathrm{m}^{2}$.dia) e armazenadas em câmaras nas temperaturas de $0{ }^{\circ} \mathrm{C}$ (controle), $25{ }^{\circ} \mathrm{C}$ (ambiente) e $35{ }^{\circ} \mathrm{C}$ (condição acelerada), com base na proposição de VITALI et al. [17] para realização de Testes Acelerados de Vida-de-Prateleira (TAVP), utilizando alimentos secos e de umidade intermediária. As câmaras de armazenamento não possuíam controle de umidade relativa. O período de estudo foi de 120 dias, sendo a primeira amostragem realizada no $15^{\circ}$ dia e as seguintes a cada 20 dias.

Foram realizadas as seguintes análises na fruta estruturada e desidratada:

\subsection{1 - Análises físico-químicas}

pH [5]; acidez [5]; atividade de água experimental medida em higrômetro eletrônico digital (Aqualab, Decagon Devices Inc., EUA) e corrigida utilizando-se a equação de correção obtida da calibração do equipamento com soluções de atividade de água conhecida; firmeza medida em texturômetro TA.XT2 da Stable Micro Systems (Godalming, EUA), utilizando sonda cilíndrica de $35 \mathrm{~mm}$ de diâmetro, denominada P/35 e célula de carga de $25 \mathrm{~kg}$, conforme metodologia descrita no manual de aplicações do TA.XT2 referência SWTI/P35 [13].

\subsection{2 - Análise microbiológica}

Contagem de bolores e leveduras realizada pelo método de plaqueamento em superfície segundo SILVA et al. [12].

\subsection{3 - Avaliação sensorial}

A fruta estruturada foi avaliada por equipe de 12 provadores treinados, utilizando escala linear de $9 \mathrm{~cm}$, quanto à aparência (ruim a excelente), gosto (característico a estranho), textura (pouco a muito firme) e qualidade global (ruim a excelente), segundo metodologia descrita em FARIA e YOTSUYANAGI [2]. Os escores dos atributos sensoriais foram analisados pela Análise de Variância (ANOVA) e teste de Tukey, para comparação entre as médias dos atributos. $\mathrm{O}$ fim da vida útil da fruta estruturada foi estabelecido quando o produto atingiu a nota 4,5 (não gostei/nem desgostei) da escala de qualidade global do teste sensorial.

\section{3 - RESULTADOS E DISCUSSÃO}

\section{1 - Acompanhamento da vida-de-prateleira}

\subsection{1 - pH e acidez}

$\mathrm{O}$ pH da fruta estruturada praticamente não variou, mantendo-se em torno de 4,5 nas três condições de armazenamento. A acidez titulável, por conseguinte, também não se alterou, mantendo-se o valor inicial de 0,56 g ácido cítrico/100 g de amostra durante o período estudado.

\subsection{2 - Atividade de água e umidade}

As amostras armazenadas a $0{ }^{\circ} \mathrm{C}$ mantiveram constantes os valores iniciais de atividade de água $(0,605)$ e umidade ( $12 \%$ ) durante o período de estudo, indicando que a embalagem utilizada protegeu o material das condições externas. A $35{ }^{\circ} \mathrm{C}$ foram observadas maiores variações, tanto na umidade quanto na atividade de água, com reduções em torno de $60 \%$ e $20 \%$, respectivamente, nos teores iniciais, indicando a influência da temperatura e, provavelmente, da umidade relativa da câmara nesses parâmetros. Nas amostras armazenadas à temperatura ambiente $\left(25^{\circ} \mathrm{C}\right)$, foi observada queda linear na umidade, com redução em $30 \%$ no teor inicial após 120 dias. Entretanto, essa diminuição não foi suficiente para alterar significativamente a atividade de água da amostra, que variou no mesmo período de 0,605 para 0,590. A diminuição na umidade do produto armazenado a temperaturas mais elevadas pode ser explicada com base na transferência de massa do alimento para a atmosfera que o envolve, que depende do teor de umidade, composição do alimento, temperatura e umidade do ar [14]. A força motriz para esse intercâmbio é dada pela diferença entre a pressão parcial de vapor d'água dos componentes, a umidade migrando do componente de maior pressão parcial de vapor ou atividade de água para o de menor pressão parcial de vapor ou atividade de água, e o equilíbrio sendo atingido num valor intermediário de atividade de água [14]. De acordo com QUAST [11], o equilíbrio é sempre o mesmo, mas o tempo para atingi-lo é diferente.

\subsection{3 - Firmeza objetiva}

De forma geral, os produtos apresentaram comportamento similar em relação à firmeza (Figura 1). Porém, a temperatura de armazenamento mais elevada $\left(35^{\circ} \mathrm{C}\right)$ conferiu maior aumento na firmeza dos produtos estruturados durante o período estudado. A provável explicação para este fato pode estar relacionada com a perda de umidade resultante de alterações na matriz do gel, composta de polpa de mamão, açúcar e hidrocolóides (alginato + pectina

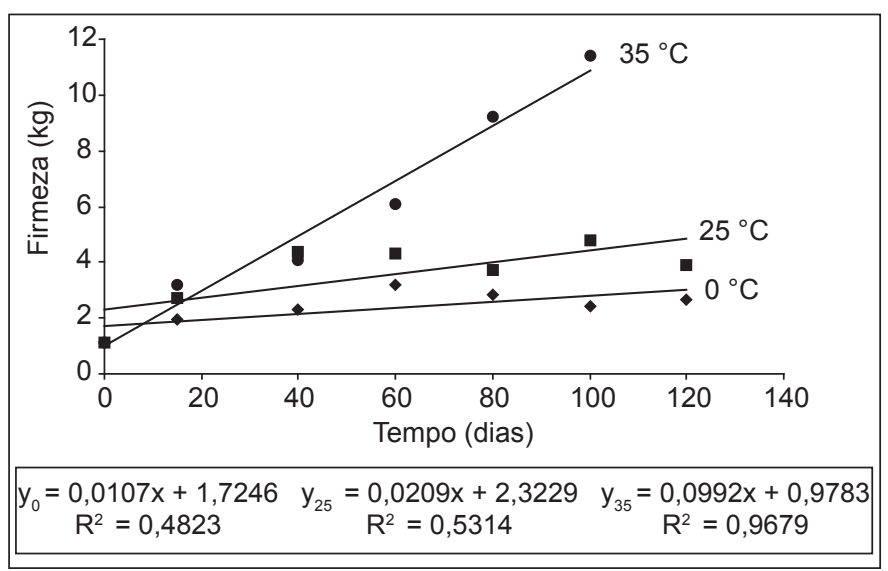

FIGURA 1 - Modelo cinético da firmeza objetiva da fruta estruturada e desidratada, armazenada a $0{ }^{\circ} \mathrm{C}, 25{ }^{\circ} \mathrm{C}$ e $35{ }^{\circ} \mathrm{C}$ durante 120 dias e respectivos ajustes lineares 
baixa metoxilação), influenciada pela elevada temperatura de armazenamento $\left(35^{\circ} \mathrm{C}\right)$.

A partir da equação de regressão linear $\left(\mathrm{y}_{0}, \mathrm{y}_{25}\right.$ ou $\left.\mathrm{y}_{35}\right)$, é possível calcular valores de firmeza (em kg) que, correlacionados com as alterações sensoriais, podem ser usados como indicativos do fim da vida útil da fruta estruturada. Um exemplo desse cálculo pode ser obtido da Figura 1. Com base no limite de vida útil estabelecido em 56 dias para o produto a $35{ }^{\circ} \mathrm{C}$ no Teste Acelerado de Vida-de-Prateleira, TAVP, e na curva de regressão linear para firmeza na mesma temperatura, $\mathrm{y}_{35}=0,0992+0,9783, \mathrm{R}^{2}=96,79 \%$, obtevese $6,5 \mathrm{~kg}$ de firmeza para fruta estruturada, que pode ser considerado um valor de referência em testes de rotina para predizer o fim da vida útil da fruta estruturada.

\subsection{4 - Avaliação microbiológica}

A fruta estruturada apresentou boa estabilidade microbiológica, visto que, no período estudado não foi observado qualquer crescimento microbiano.

\subsection{5 - Avaliação sensorial}

Nas Tabelas $2 a, 2 b, 2 c$ e $2 d$, são apresentadas, respectivamente, as médias dos atributos sensoriais: aparência, gosto, textura e qualidade global, atribuídas pela equipe de 12 provadores treinados, às amostras de fruta estruturada armazenadas a 0,25 e $35{ }^{\circ} \mathrm{C}$, durante 120 dias. Os resultados obtidos mostraram que a qualidade global foi o atributo sensorial que melhor representou a perda na qualidade da fruta estruturada durante a estocagem, provavelmente resultado da avaliação conjunta da aparência e da firmeza. O modelo cinético da qualidade global é mostrado na Figura 2, dada a sua importância. De acordo com NETO [10] o termo "qualidade" é usado como uma forma de expressão do estado de deterioração do alimento, e significa, para o consumidor, que o alimento possui características ou atributos desejáveis. Os provadores não perceberam diferenças

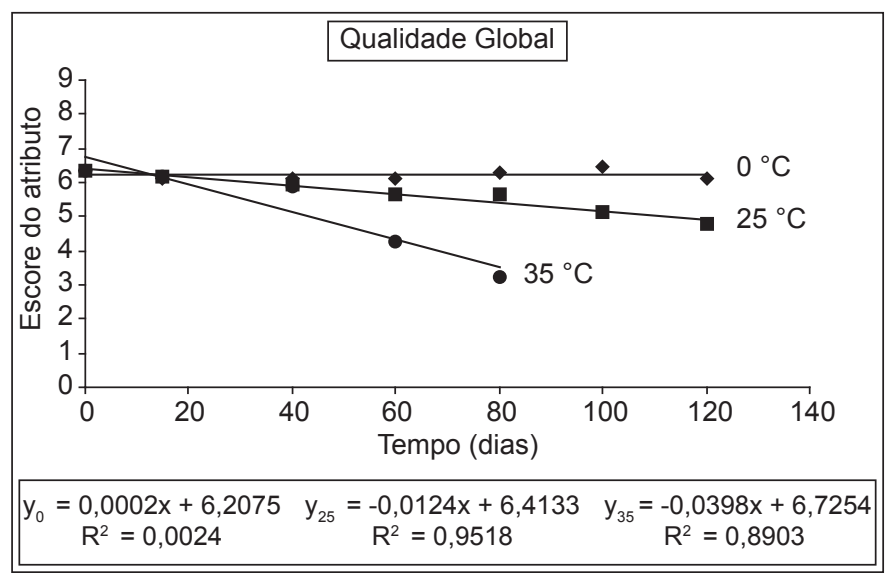

FIGURA 2 - Modelo cinético da qualidade global da fruta estruturada e desidratada, armazenada a $0{ }^{\circ} \mathrm{C}, 25^{\circ} \mathrm{C}$ e $35{ }^{\circ} \mathrm{C}$ durante 120 dias, e respectivos ajustes lineares.
TABELA 2A - Média dos escores de aparência ${ }^{1}$ da fruta estruturada armazenada sob três condições de temperatura.

\begin{tabular}{cccccccc}
\hline $\begin{array}{c}\text { Temperatura } \\
\text { de armazena- } \\
\text { mento }\left({ }^{\circ} \mathrm{C}\right)\end{array}$ & $\mathbf{0}$ & $\mathbf{1 5}$ & $\mathbf{4 0}$ & $\mathbf{6 0}$ & $\mathbf{8 0}$ & $\mathbf{1 0 0}$ & $\mathbf{1 2 0}$ \\
\hline 0 & $6,25^{\mathrm{a}, \mathrm{A}}$ & $5,86^{\mathrm{a}, \mathrm{A}}$ & $6,38^{\mathrm{a}, \mathrm{A}}$ & $6,36^{\mathrm{a}, \mathrm{A}}$ & $5,84^{\mathrm{a}, \mathrm{A}}$ & $6,19^{\mathrm{a}, \mathrm{A}}$ & $5,37^{\mathrm{a}, \mathrm{A}}$ \\
25 & $6,25^{\mathrm{a}, \mathrm{A}}$ & $6,04^{\mathrm{a}, \mathrm{A}}$ & $6,08^{\mathrm{a}, \mathrm{A}}$ & $5,57^{\mathrm{ab}, \mathrm{A}}$ & $5,10^{\mathrm{a}, \mathrm{A}}$ & $4,82^{\mathrm{a}, \mathrm{A}}$ & $4,84^{\mathrm{a}, \mathrm{A}}$ \\
35 & $6,25^{\mathrm{a}, \mathrm{A}}$ & $6,16^{\mathrm{a}, \mathrm{A}}$ & $5,86^{\mathrm{a}, \mathrm{A}}$ & $4,58^{\mathrm{b}, \mathrm{A}}$ & $4,82^{\mathrm{a}, \mathrm{A}}$ & n.d. & n.d.
\end{tabular}

Médias com letras minúsculas iguais na mesma coluna, não há diferença significativa ao nível de 95\% de probabilidade; Médias com letras maiúsculas iguais na mesma linha, não há diferença significativa ao nível de $95 \%$ de probabilidade; e ${ }^{1}$ Escores de aparência entre os extremos inferior ( 1 = ruim) e superior $(9=$ excelente).

TABELA 2B - Média dos escores de gosto ${ }^{1}$ da fruta estruturada armazenada sob três condições de temperatura.

\begin{tabular}{cccccccc}
\hline $\begin{array}{c}\text { Temperatura } \\
\text { de armazena- } \\
\text { mento }\left({ }^{\circ} \mathrm{C}\right)\end{array}$ & $\mathbf{0}$ & $\mathbf{1 5}$ & $\mathbf{4 0}$ & $\mathbf{6 0}$ & $\mathbf{8 0}$ & 100 & 120 \\
\hline 0 & $6,32^{\mathrm{a}, \mathrm{A}}$ & $7,07^{\mathrm{a}, \mathrm{A}}$ & $6,90^{\mathrm{a}, \mathrm{A}}$ & $6,48^{\mathrm{a}, \mathrm{A}}$ & $6,23^{\mathrm{a}, \mathrm{A}}$ & $6,51^{\mathrm{a}, \mathrm{A}}$ & $5,69^{\mathrm{a}, \mathrm{A}}$ \\
25 & $6,32^{\mathrm{a}, \mathrm{A}}$ & $7,09^{\mathrm{a}, \mathrm{A}}$ & $6,76^{\mathrm{a}, \mathrm{A}}$ & $6,34^{\mathrm{a}, \mathrm{A}}$ & $6,39^{\mathrm{a}, \mathrm{A}}$ & $6,01^{\mathrm{a}, \mathrm{A}}$ & $5,45^{\mathrm{a}, \mathrm{A}}$ \\
35 & $6,32^{\mathrm{a}, \mathrm{A}}$ & $7,05^{\mathrm{a}, \mathrm{A}}$ & $6,58^{\mathrm{a}, \mathrm{A}}$ & $5,64^{\mathrm{a}, \mathrm{A}}$ & $4,94^{\mathrm{a}, \mathrm{A}}$ & n.d. & n.d.
\end{tabular}

Médias com letras minúsculas iguais na mesma coluna, não há diferença significativa ao nível de 95\% de probabilidade; Médias com letras maiúsculas iguais na mesma linha, não há diferença significativa ao nível de $95 \%$ de probabilidade; e ${ }^{1}$ Escores de gosto entre os extremos inferior ( 1 = estranho) e superior $(9$ = característico).

TABELA 2C - Média dos escores de textura ${ }^{1}$ da fruta estruturada armazenada sob três condições de temperatura.

\begin{tabular}{cccccccc}
\hline $\begin{array}{c}\text { Temperatura } \\
\text { de armazena- } \\
\text { mento }\left({ }^{\circ} \mathrm{C}\right)\end{array}$ & $\mathbf{0}$ & $\mathbf{1 5}$ & $\mathbf{4 0}$ & $\mathbf{6 0}$ & $\mathbf{8 0}$ & $\mathbf{1 0 0}$ & $\mathbf{1 2 0}$ \\
\hline 0 & $5,77^{\mathrm{a}, \mathrm{A}}$ & $6,45^{\mathrm{a}, \mathrm{A}}$ & $5,41^{\mathrm{b}, \mathrm{A}}$ & $5,61^{\mathrm{b}, \mathrm{A}}$ & $4,86^{\mathrm{b}, \mathrm{A}}$ & $5,11^{\mathrm{b}, \mathrm{A}}$ & $5,32^{\mathrm{a}, \mathrm{A}}$ \\
25 & $5,77^{\mathrm{a}, \mathrm{AB}}$ & $6,54^{\mathrm{a}, \mathrm{A}}$ & $5,82^{\mathrm{a}, \mathrm{AB}}$ & $4,91^{\mathrm{b}, \mathrm{B}}$ & $4,95^{\mathrm{b}, \mathrm{B}}$ & $6,17^{\mathrm{b}, \mathrm{AB}}$ & $6,00^{\mathrm{a}, \mathrm{AB}}$ \\
35 & $5,77^{\mathrm{a}, \mathrm{C}}$ & $6,15^{\mathrm{a}, \mathrm{C}}$ & $6,83^{\mathrm{a}, \mathrm{BC}}$ & $7,58^{\mathrm{a}, \mathrm{AB}}$ & $8,08^{\mathrm{a}, \mathrm{A}}$ & n.d. & n.d. \\
\hline
\end{tabular}

Médias com letras minúsculas iguais na mesma coluna, não há diferença significativa ao nível de 95\% de probabilidade: Médias com letras maiúsculas iguais na mesma linha, não há diferença significativa ao nível de $95 \%$ de probabilidade; e ${ }^{1}$ Escores de textura entre os extremos inferior ( 1 = pouco firme) e superior $(9=$ muito firme $)$.

TABELA 2D - Média dos escores de qualidade global ${ }^{1}$ da fruta estruturada atribuída por equipe de provadores treinados armazenada sob três condições de temperatura.

\begin{tabular}{cccccccc}
\hline $\begin{array}{c}\text { Temperatura } \\
\text { de armazena- } \\
\text { mento }\left({ }^{\circ} \mathrm{C}\right)\end{array}$ & $\mathbf{0}$ & $\mathbf{1 5}$ & $\mathbf{4 0}$ & $\mathbf{6 0}$ & $\mathbf{8 0}$ & $\mathbf{1 0 0}$ & 120 \\
\hline 0 & $6,35^{\mathrm{a}, \mathrm{A}}$ & $6,12^{\mathrm{a}, \mathrm{A}}$ & $6,09^{\mathrm{a}, \mathrm{A}}$ & $6,10^{\mathrm{a}, \mathrm{A}}$ & $6,31^{\mathrm{a}, \mathrm{A}}$ & $6,44^{\mathrm{a}, \mathrm{A}}$ & $5,94^{\mathrm{a}, \mathrm{A}}$ \\
25 & $6,35^{\mathrm{a}, \mathrm{A}}$ & $6,18^{\mathrm{a}, \mathrm{A}}$ & $5,97^{\mathrm{a}, \mathrm{A}}$ & $5,65^{\mathrm{a}, \mathrm{A}}$ & $5,66^{\mathrm{a}, \mathrm{A}}$ & $5,11^{\mathrm{a}, \mathrm{A}}$ & $4,85^{\mathrm{a}, \mathrm{A}}$ \\
35 & $6,35^{\mathrm{a}, \mathrm{A}}$ & $6,15^{\mathrm{a}, \mathrm{A}}$ & $5,89^{\mathrm{a}, \mathrm{A}}$ & $4,24^{\mathrm{a}, \mathrm{AB}}$ & $3,22^{\mathrm{b}, \mathrm{B}}$ & n.d. & n.d.
\end{tabular}

Médias com letras minúsculas iguais na mesma coluna, não há diferença significativa ao nível de 95\% de probabilidade; Médias com letras maiúsculas iguais na mesma linha, não há diferença significativa ao nível de $95 \%$ de probabilidade; e ${ }^{1}$ Escores de qualidade global entre os extremos inferior ( $1=$ ruim) e superior ( 9 = excelente). 
significativas $(p \leq 0,05)$ no gosto e na aparência da fruta estruturada durante o período de avaliação. Visualmente, foi observado ligeiro escurecimento na amostra armazenada a $35^{\circ} \mathrm{C}$, a partir do $60^{\circ}$ dia de armazenamento, de acordo com observações dos provadores. No entanto, a aparência não foi comprometida, provavelmente devido à cobertura de açúcar impalpável. A textura manteve-se inalterada nos produtos mantidos a 0 e $25^{\circ} \mathrm{C}$ durante o período de avaliação. $\mathrm{O}$ aumento na dureza foi significativo $(\mathrm{p} \leq 0,05)$ apenas nas amostras a $35^{\circ} \mathrm{C}$, a partir do $60^{\circ}$ dia de armazenamento, e pode ser considerado o principal mecanismo de deterioração responsável pelo final da vida-de-prateleira do produto armazenado na condição acelerada.

Adotando-se como perda de qualidade máxima aceitável o valor 4,5 (não gostei/nem desgostei) da escala de qualidade global do teste sensorial, determinou-se a vida útil em 56 dias do produto armazenado na condição acelerada $\left(35^{\circ} \mathrm{C}\right)$. A fruta estruturada armazenada a $35^{\circ} \mathrm{C}$ foi excluída dos testes sensoriais a partir do $100^{\circ}$ dia de armazenamento, porque os provadores atribuíram notas abaixo do valor estabelecido para o fim da vida útil, sendo consideradas inaceitáveis.

A partir das equações de regressão linear $\left(\mathrm{y}_{0}, \mathrm{y}_{25}\right.$ e $\left.\mathrm{y}_{35}\right)$ obtidas do modelo cinético da qualidade global, Figura 2 , obtiveram-se as constantes da velocidade de reação $(k)$ para as três temperaturas de armazenamento. Foi construído o gráfico, em escala linear, plotando-se o valor do logaritmo das constantes de velocidade de reação (ln k), na ordenada, em função do inverso da temperatura absoluta (1/T), na abcissa. A reta obtida, $y=-10012 x+29,239$, com coeficiente de correlação linear $\left(\mathrm{R}^{2}\right)$ igual a 99,94\%, indicou que essa equação segue o modelo de Arrhenius [6]. Os parâmetros cinéticos mostrados na Tabela 3 , energia de ativação $\left(\mathrm{E}_{\mathrm{a}}\right)$ igual a $19,89 \mathrm{kcal} / \mathrm{mol} . \mathrm{K}$, e o valor de $Q_{10}$ igual a 3 , são compatíveis com os valores para qualidade sensorial global de produtos de origem vegetal, citado por TEIXEIRA NETTO et al.[15]. O TAVP permitiu estabelecer a vida útil do produto em 56 dias, na condição acelerada. Com base no tempo de vida útil (56 dias) e do fator de aceleração da temperatura $\left(Q_{10}\right)$, foi possível estimar a vida-de-prateleira do produto armazenado a $25{ }^{\circ} \mathrm{C}$ em 168 dias. Esse resultado encontra-se próximo dos 154 dias estimados com base na equação de regressão linear $\left(\mathrm{y}_{25}=-0,0121 \mathrm{x}+6,4045 ; \mathrm{R}^{2}=95,18 \%\right)$ da qualidade global, do teste sensorial. De fato, no teste em tempo real, em condições normais de armazenamento $\left(25^{\circ} \mathrm{C}\right)$, o produto com 120 dias de armazenamento apresentou média 4,85 , acima do valor estabelecido para determinar o fim da vida útil e, portanto, adequado para o consumo.

TABELA 3 - Parâmetros cinéticos da qualidade global da fruta estruturada.

\begin{tabular}{lccc}
\hline Parâmetros cinéticos & \multicolumn{3}{c}{ Temperatura $\left({ }^{\circ} \mathbf{C}\right)$} \\
\cline { 2 - 4 } & $\mathbf{0}$ & $\mathbf{2 5}$ & $\mathbf{3 5}$ \\
\hline $\mathrm{k}\left(\mathrm{dia}^{-1}\right)$ & $2 \mathrm{E}-04$ & $1,24 \mathrm{E}-02$ & $3,98 \mathrm{E}-02$ \\
$\mathrm{r}^{2}(\%)$ & - & 95,18 & 89,03 \\
$\mathrm{Ea}(\mathrm{kcal} / \mathrm{mol})$ & - & 19,89 & - \\
$\mathrm{r}^{2}(\%)$ & - & 99,94 & - \\
$\mathrm{Q}_{10}$ & - & 3 & - \\
\hline
\end{tabular}

\section{4 - CONCLUSÕES}

O desenvolvimento deste trabalho possibilitou as seguintes conclusões:

- A qualidade global sensorial e a textura objetiva foram os atributos de maior importância no monitoramento da perda de qualidade da fruta estruturada de polpa concentrada de mamão;

- O valor 6,5 kg de firmeza para fruta estruturada pode ser utilizado como referência para estabelecer o fim da vida útil da fruta estruturada em testes de rotina;

- A fruta estruturada e desidratada (12\% de umidade, $\mathrm{Aa}=0,605)$ manteve-se microbiologicamente estável nas temperaturas de $0{ }^{\circ} \mathrm{C}, 25{ }^{\circ} \mathrm{C}$ e $35{ }^{\circ} \mathrm{C}$, durante o período de 120 dias de armazenamento;

- Com base no teste acelerado de vida-de-prateleira (TAVP) a vida-de-prateleira do produto armazenado a $25{ }^{\circ} \mathrm{C}$ foi estimada em 168 dias; e

- O teste em tempo real, em condições normais de armazenamento $\left(25^{\circ} \mathrm{C}\right)$, o tempo para o produto atingir o fim da vida-de-prateleira, de acordo com as condições estabelecidas neste trabalho, pode chegar a 154 dias.

\section{5 - REFERÊNCIAS BIBLIOGRÁFICAS}

[1] ANTUNES, A. J.; CANHO, V. P. Aditivos em alimentos. São Paulo:Secretaria da Indústria, Comércio, Ciência e Tecnologia. 1983. 178 p.

[2] FARIA, E. Z.; YOTSUYANAGI, K. Técnicas de análise sensorial. Campinas: ITAL. $1^{\mathrm{a}}$ ed., 2002. 116 p.

[3] GRizotTo, R. K.; AGUiRRE, J. M.; ClAUS, M. L. Estudo da concentração da polpa de mamão para produção de fruta estruturada. In: $6^{\circ} \mathrm{SIMPÓSIO}$ LATINO AMERICANO DE CIENNCIA DE ALIMENTOS. 2005, Campinas: SLACA. 1p. CD Room. 2005.

[4] GRIzOTTO, R. K.; BRUNS, R. E.; AGUIRRE, J. M; BATISTA, G. Otimização via Metodologia de Superfície de Respostas dos parâmetros tecnológicos para produção de fruta estruturada e desidratada a partir de polpa concentrada de mamão. Ciência e Tecnologia, v. 25 , n. 1, p. 158-164, 2005.

[5] HORWITZ, W (ed.). Official Methods of Analysis of AOAC International. 17 ed. Gaithersburg, Maryland, 2000.

[6] LABUZA, T. P. Application of chemical kinetics to deterioration of foods. J. Chem. Edu., v. 61, n. 4, p. 348-358, 1984.

[7] LABUZA, T. P. Shelf-life Dating of Foods. Westport: Food and Nutrition Press, 1982. 500 p.

[8] MORI, E. E. M. Determinação da vida-de-prateleira através da análise sensorial e correlações. In: REAÇÕES DE TRANSFORMAÇÃO E VIDA-DE-PRATELEIRA DE ALIMENTOS PROCESSADOS. Moura, S.C.S.R.; Germer, S.P.M. (ed.) Campinas: ITAL. $3^{\mathrm{a}}$ ed. p. 63-83, 2004. (Manual Técnico $\mathrm{n}^{\circ}$ 6).

[9] MOUgueT, C.; DUMA, J. C.; GuIlBerT, S. Texturization of sweetened mango pulp: optimization using 
response surface methodology. J. Food Sci., v. 57, n. 6, p.1395-1400, 1992.

[10] NETTO, F. M. Determinação da vida-de-prateleira - Erros e limitações. In: REAÇÕES DE TRANSFORMAÇÃO E VIDA-DE-PRATELEIRA DE ALIMENTOS PROCESSADOS. Moura, S. C. S. R.; Germer, S. P. M. Campinas: ITAL. $3^{\mathrm{a}}$ ed. p. 83-92, 2004. (Manual Técnico $\left.\mathrm{n}^{\mathrm{0}} 6\right)$.

[11] QUAST, D. Quem tem medo de atividade de água? In: ATIVIDADE DE ÁGUA EM ALIMENTOS. Jardim, D.C.P.; Germer, S. P. M. (coord.) Campinas: ITAL. p. 13-1 a 13-8, 1997.

[12] SILVA, N.; JUNQUEIRA, V. C. A.; SIlvEIRA, N. F. A. Manual de métodos de análise microbiológica de alimentos. São Paulo: Varella. $2^{\mathrm{a}}$ ed. p. 26-28, 2001.

[13] STABLE MICRO SYSTEMS. User Manual. Texture Analyser. Model TA-XT2i, Godalming, version 6.10 e 7.10, Agosto de 1997, 87p.

[14] TEIXEIRA NETO, R. O. Atividade de água e transformação dos alimentos. In: ATIVIDADE DE ÁGUA EM ALIMENTOS. Jardim, D.C.P.; Germer, S.P.M. (coord.) Campinas: ITAL. p. 2-1 a 2-9, 1997.

[15] TEIXEIRA NETO, R. O.; VITALI, A. A.; MOURA, S. C. $\mathrm{S}$. R. Introdução à cinética de reação em alimentos. In: REAÇÕES DE TRANSFORMAÇÃO E VIDA-DE-PRA-
TELEIRA DE ALIMENTOS PROCESSADOS. Moura, S. C. S. R.; Germer, S. P. M. (ed.) Campinas: ITAL. $3^{\mathrm{a}}$ ed. p. 63-83, 2004. (Manual Técnico n6).

[16] VIJAYANAND, P.; YADAV, A. R.; BALASUBRAMANYAM, N.; NARASIMHAM, P. Storage stability of guava fruit bar prepared using a new process. Lebens. Wiss. Tecnol. v. 33, n. 1, p. 132-137, 2000.

[17] VITALI, A. A.; TEIXEIRA NETO, R. O.; GERMER, S. P. M. Testes Acelerados de vida-de-prateleira de alimentos. In: REAÇÕES DE TRANSFORMAÇÃO E VIDA-DE-PRATELEIRA DE ALIMENTOS PROCESSADOS. Moura, S. C. S. R.; Germer, S. P. M. Campinas: ITAL. 3a ed. p. 75-92, 2004. (Manual Técnico no 6).

[18] WRIGHT, B.B.; TAUB, I.A. Stored product quality: Open dating asd temperature monitoring. In: FOOD STORAGE STABILITY. Taub, I. A.; Singh, R. P. (eds.). Boca Raton: CRC Press, p. 353-368, 1997.

\section{6 - AGRADECIMENTOS}

Os autores agradecem ao CNPq pela concessão de Bolsa de Iniciação Científica e ao produtor rural Sr. João Luis Bayer, na cidade de Santa Maria da Vitória (BA), pela doação das frutas. Agradecem, também, ao estudante Rafael Lourenço Thomaz Favery pela realização das análises estatísticas. 